\title{
Polarimetric sensitivity to hydrostatic pressure and temperature in birefringent dual-core microstructured polymer fiber
}

\author{
Marcin K. Szczurowski ${ }^{1}$, Tadeusz Martynkien ${ }^{1}$, Gabriela Statkiewicz-Barabach ${ }^{1}$, Waclaw \\ Urbanczyk $^{1}$, David J. Webb ${ }^{2}$ \\ ${ }^{1}$ Institute of Physics, Wroclaw University of Technology, Wybrzeze Wyspianskiego 27, 50-370 \\ Wroclaw, Poland \\ ${ }^{2}$ Photonics Research Group, Aston University, Birmingham, B4 7ET, UK
}

\begin{abstract}
We experimentally characterized a birefringent microstructured polymer fiber of specific construction, which allows for single mode propagation in two cores separated by a pair of large holes. The fiber exhibits high birefringence in each of the cores as well as relatively weak coupling between the cores. Spectral dependence of the group and the phase modal birefringence was measured using an interferometric method. We have also measured the sensing characteristics of the fiber such as the polarimetric sensitivity to hydrostatic pressure and temperature.
\end{abstract}

Keywords: polymer microstructured fibers, birefringence, sensitivity to pressure and temperature.

\section{INTRODUCTION}

In the last few years, interest in sensing applications of polymer optical fibers (POF) significantly increased. It is driven by the fact that POF posses several advantages compared to silica fibers. In particular, POF are biocompatible, offer greater numerical aperture, more flexibility (lower Young's modulus) and can be exposed to much greater strain compared to silica. For these reasons, conventional POF have been already studied in numerous sensing applications including measurements of large strains [1-2], humidity [3], and $\mathrm{pH}$ [4]. The inscription of Bragg gratings in POF, first demonstrated in [5], opens now new sensing possibilities [6]. In the last decade the fabrication technology of microstructured polymer optical fibers ( $\mathrm{mPOF}$ ) was mastered by several groups and $\mathrm{mPOF}$ of many different types have been already reported in the literature. This new class of fibers combines the interesting physical and chemical properties of PMMA with the wide engineering freedom of microstructured fibers. The possibility of fabrication of Bragg gratings in mPOF was also demonstrated [7] and several sensing applications of such elements have been already studied. To our knowledge, so far there are no publications in the literature on the sensing characteristics of birefringent mPOFs. In this paper, we present the results of experimental investigations of highly birefringent dual-core mPOF and report on the polarimetric sensitivity of this fiber to hydrostatic pressure and temperature.

\section{INVESTIGATED MICROSTRUCTURED POLYMER FIBER}

The investigated mPOF was purchased from Kyriama Pty Ltd. of Sydney, Australia. A SEM image of the fiber crosssection is shown in Fig.1. In the microstructured region of this fiber there are two very large holes and a tiny bridge of PMMA between them. This bridge is so narrow that it does not permit light propagation. As a result, the modes are localized at both ends of the bridge where its thickness is greater. Both these cores are single mode and possess high birefringence induced by lack of hexagonal symmetry in their surroundings. Because of the low distance between the cores a coupling effect is observed. In such a case, light is guided in the form of orthogonally polarized even and odd supermodes (respectively $\mathrm{S}_{\mathrm{e}}{ }^{\mathrm{x}}, \mathrm{S}_{\mathrm{e}}{ }^{\mathrm{y}}, \mathrm{S}_{\mathrm{o}}{ }^{\mathrm{x}}, \mathrm{S}_{\mathrm{o}}{ }^{\mathrm{y}}$ ) spreading simultaneously over both cores.

The coupling effect is clearly visible in the investigated fiber. We have observed interference fringes in the spectrograms registered by collecting the light from the excited core on the slit of a miniature spectrometer. The spectrograms were registered for $\mathrm{x}$ - and y-polarizations. The contrast of interference fringes arising due to intermodal interference is about 0.6, which corresponds to power division between dominant and weak supermodes in the ratio of about 90:10. The estimated power division between the supermodes confined in the excited core leads to the conclusion that relatively weak coupling takes place in the investigated fiber. This is also in agreement with the results of numerical simulations obtained for the actual fiber geometry. We have observed a very high impact of the fiber bending on the coupling effect. Therefore, in all measurements reported in this communication, care was taken not to bend the fiber when the applied measurand was changed.

Fourth European Workshop on Optical Fibre Sensors, edited by José Luís Santos, Brian Culshaw, José Miguel López-Higuera, William N. MacPherson, Proc. of SPIE Vol. 7653, 76530D

(c) 2010 SPIE · CCC code: $0277-786 X / 10 / \$ 18 \cdot$ doi: $10.1117 / 12.865856$

Proc. of SPIE Vol. 7653 76530D-1 


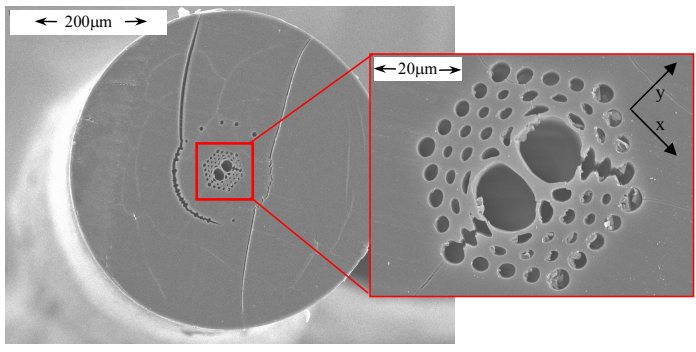

Core 1 , supermode $\mathrm{S}_{\mathrm{e}}{ }^{\mathrm{x}}$
Core 2

supermode $\mathrm{S}_{0}{ }^{\mathrm{x}}$

Fig.1. SEM image of the investigated mPOF and calculated amplitude distribution in x-polarized even and odd supermodes.

\section{MESUREMENT RESULTS}

The group modal birefringence in both cores was measured using a spectral interferometric method. The measurement set-up consisted of a broad band light source (supercontinuum), polarizer and analyzer placed at the ends of the tested fiber and aligned at $45^{\circ}$ to its polarization axes. Such alignment causes pairs of orthogonally polarized supermodes to be excited in the fiber which interfere at its output after passing through the analyzer. A microscope objective formed a sharp image of the fiber end-face on the entrance slit of the spectrometer. In this way, we could register spectral interference fringes individually for each core. With this arrangement, because of weak coupling between the cores, we could measure the birefringence and sensitivity in each core individually. To determine the spectral dependence of the phase modal birefringence B from the registered spectrograms, we first measured a beat length using the lateral force method The obtained values at $\lambda=610 \mathrm{~nm}$ were $7.75 \mathrm{~mm}$ and $7.61 \mathrm{~mm}$, respectively, for the first and the second core. In the second step, we calculated the phase modal birefringence $B$ at $\lambda=610 \mathrm{~nm}$ and the absolute value of the phase shift between polarization modes at this wavelength. When the absolute interference order is know at one wavelength, it is possible to determine the phase shift $\Delta \varphi(\lambda)$ at each intensity extreme in the registered spectrograms and finally to calculate the spectral dependence of the phase modal birefringence. The group modal birefringence was determined for both cores from the following relation:

$$
G(\lambda)=-\frac{\lambda^{2}}{2 \pi L} \frac{d(\Delta \varphi(\lambda))}{d \lambda}
$$

Birefringence in both cores strongly increases with wavelength and significantly exceeds $10^{-4}$ in the visible range, Fig.2. This is a typical behavior of $\mathrm{B}$ and $\mathrm{G}$ for microstructured fibers with purely geometrical birefringence. Because both cores have similar shapes and sizes, the measured values of B and $\mathrm{G}$ differ only a little between the cores (a maximum of $10 \%)$.

The polarimetric sensitivity of the fiber to a specific parameter, $\mathrm{X}$, represents the phase difference between polarization modes induced by unit change of the parameter over unit length of the fiber and can be expressed in the following way:

$$
K_{X}=\frac{1}{L_{X}} \frac{d\left(\phi_{x}-\phi_{y}\right)}{d X}=\frac{2 \pi}{\lambda}\left[\frac{\partial B}{\partial X}+\frac{1}{L_{X}} \frac{\partial L_{X}}{\partial X}\right] .
$$

To determine the $\mathrm{K}_{\mathrm{X}}$, the shift of spectral interference fringes was recorded as a function of applied measurand. By registering the displacement of interference minima $\lambda_{\min }(\mathrm{X})$ versus measurand change, we determined the polarimetric sensitivity from the following relation:

$$
K_{X}=-\frac{2 \pi}{L_{X}} \frac{d}{d X}\left(\frac{\lambda_{\min }(X)}{\Delta \lambda}\right)
$$

where $\Delta \lambda$ is the fringe spacing and $\mathrm{L}_{\mathrm{X}}$ is the length of the fiber exposed to measurand change. One should note that displacement of the interference fringes towards shorter wavelengths induced by increasing measurand corresponds to a positive sign of $\mathrm{K}_{\mathrm{X}}$. By aligning the input polarizer in parallel to one of the fiber symmetry axes, we could also selectively excite the supermodes of the same polarization and measure the intermodal phase sensitivity named by $K_{X}^{\text {int }-x}$ and $K_{X}^{\text {int }-y}$, respectively for pairs of supermodes of $\mathrm{x}$ - and y-polarization. The results of measurements of the polarimetric and intermodal sensitivity to pressure in core 1 are presented in Fig.3. The investigated fiber shows a repeatable response to hydrostatic pressure with small hysteresis in the investigated pressure range up to $2 \mathrm{MPa}(20 \mathrm{bars})$ and relatively high polarimetric sensitivity up to $K_{P}=72 \mathrm{rad} /(\mathrm{MPa} \times \mathrm{m})$ at $\lambda=710 \mathrm{~nm}$. The polarimetric sensitivity measured for core 2 is only 

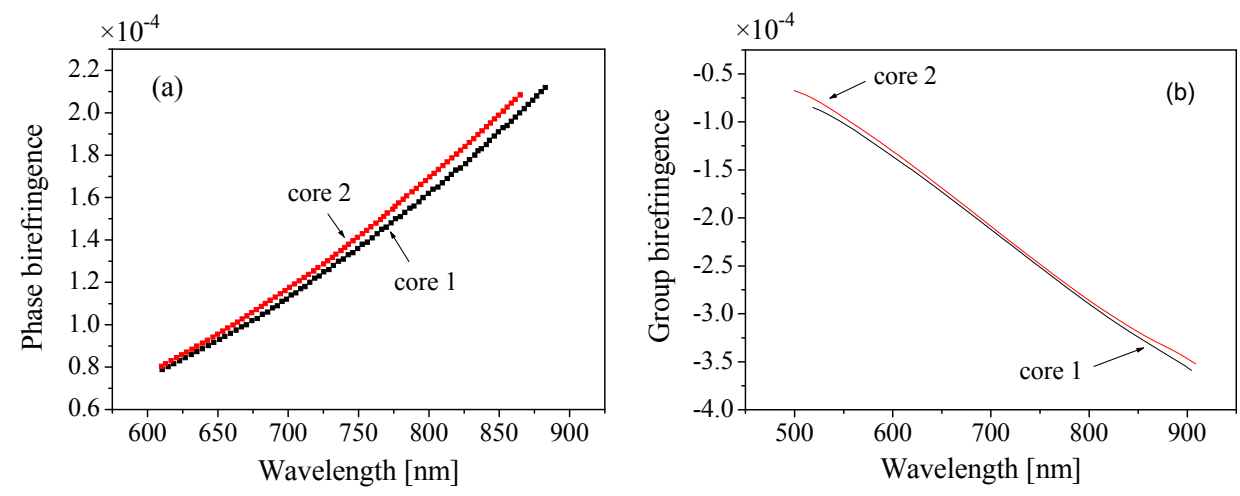

Fig.2. Results of measurements of phase modal birefringence (a) and group modal birefringence (b) in the two cores.

5\% greater than that of core 1. In Fig.3a, we also present the effect of hydrostatic pressure on the interference of the supermodes of y-polarization. An increase of the phase shift between the supermodes is observed in response to applied pressure, with the rate of about $K_{P}^{\text {int }-y}=13.5 \mathrm{rad} /(\mathrm{MPa} \times \mathrm{m})$. The value of $K_{P}^{\text {int }-x}$ is the same within the measurement precision of about $3 \%$.

The measured polarimetric sensitivities $K_{P}$ have positive sign, which means that birefringence increases in response to applied pressure. The measurement results were compared with numerical simulations of the polarimetric sensitivity to pressure carried out in the same way as done recently for microstructured silica fiber [8]. According to this analysis, as is the case in silica fibers, the sensitivity $\mathrm{dB} / \mathrm{dp}$ in $\mathrm{mPOF}$ is mostly associated with the material birefringence induced in the core region by applied pressure. As reported in our earlier publication [8], the $K_{P}$ in silica PCFs has a negative sign, however its absolute value is close to the sensitivity of the investigated mPOF. For example, the Kp reported in [9] for the birefringent microstructured silica fibers ranges from $-14.8 \mathrm{rad} / \mathrm{MPa} \times \mathrm{m}$ up to $-100 \mathrm{rad} / \mathrm{MPa} \times \mathrm{m}$ (at $830 \mathrm{~nm}$ ) depending on the fiber construction. The different signs of $\mathrm{K}_{\mathrm{P}}$ in silica and PMMA microstructured fibers is related to different signs of the stress-optic coefficients in these two materials, which are equal to $\Delta \mathrm{C}_{\mathrm{SiO} 2}=3.52 \times 10^{-12} \mathrm{~Pa}^{-1}[10]$ and $\Delta \mathrm{C}_{\mathrm{PMMA}}=-2.31 \times 10^{-12} \mathrm{~Pa}^{-1}[11]$, respectively.

Measurement of the polarimetric sensitivity to temperature was conducted on several pieces of the investigated fiber in the temperature range of $3-60^{\circ} \mathrm{C}$. Depending on the thermal history of the specific piece of the fiber, we observed very
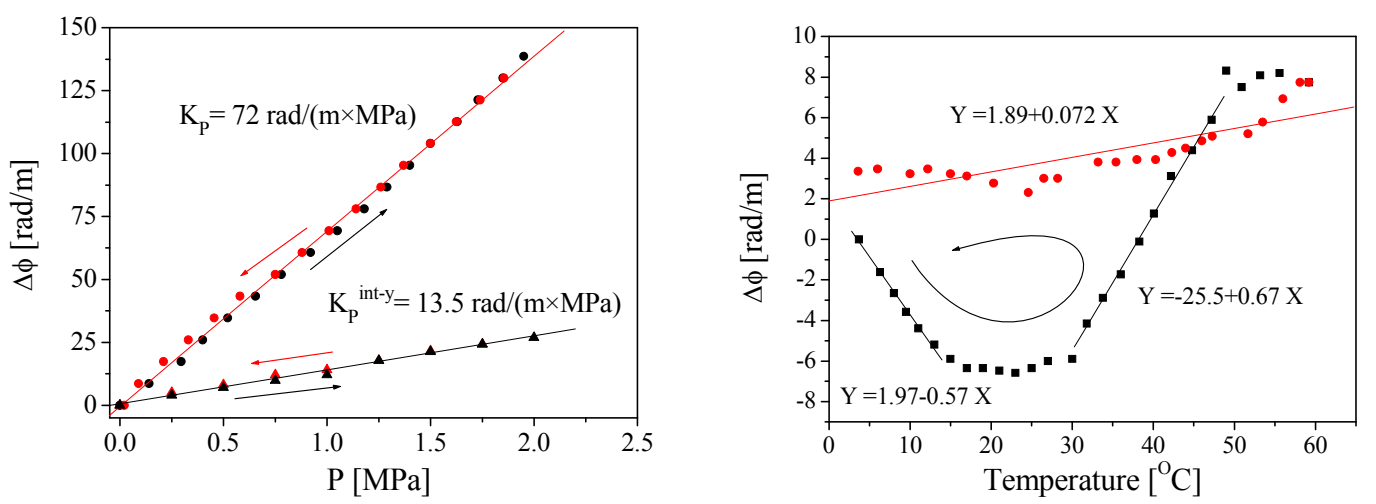

Fig.3. (a) Phase shift between polarization supermodes $\left(K_{\mathrm{P}}\right)$ and supermodes of y-polarization $\left(K_{P}^{\text {int }-y}\right)$ induced by increasing and decreasing hydrostatic pressure, $\lambda=710 \mathrm{~nm}$, length of the fiber exposed to pressure changes is $\mathrm{L}_{\mathrm{P}}=0.362 \mathrm{~m}$. (b) Phase shift between polarization supermodes induced by increasing and decreasing temperature measured for annealed fiber at $\lambda=710 \mathrm{~nm}$, length of the fiber subjected to temperature changes is $\mathrm{L}_{\mathrm{T}}=0.22 \mathrm{~m}$, both measurements were carried out for core 1 . 
different polarimetric responses to temperature, typically with high nonlinearity and hysteresis. The fiber sensitivity $K_{T}$ averaged over the full temperature span changes from $-1 \mathrm{rad} / \mathrm{K} \times \mathrm{m}$ to $1 \mathrm{rad} / \mathrm{K} \times \mathrm{m}$ depending on the temperature cycle. The origin of such large differences in $K_{T}$ between successive temperature cycles is not clear. Perhaps they are caused by release of frozen-in stress at increased temperatures. In Fig.3b, we present the temperature response of a fiber that was annealed for $20 \mathrm{~h}$ at a temperature of $100^{\circ} \mathrm{C}$. We observed that annealing improves repeatability and decreases the amplitude of the temperature response, however, nonlinearity and hysteresis is still observed. For annealed fiber the local temperature coefficients change from $-0.6 \mathrm{rad} / \mathrm{K} \times \mathrm{m}$ to $0.7 \mathrm{rad} / \mathrm{K} \times \mathrm{m}$.

\section{CONCLUSIONS}

To our knowledge, we report for the first time on the sensing characteristics of highly birefringent microstructured polymer fiber. The investigated fiber possesses two cores, with high birefringence in each core exceeding $10^{-4}$ in the visible range and shows a spectral dependence of $\mathrm{B}$ and $\mathrm{G}$ typical for geometrical birefringence. Because of small coupling between the cores, we could characterize each core individually. Small differences between the cores (of the order of a few \%) were observed for all investigated parameters. Two large holes in the microstructured region of the investigated fiber are responsible for its high polarimetric sensitivity to hydrostatic pressure, reaching $\mathrm{K}_{\mathrm{P}}=72 \mathrm{rad} /(\mathrm{MPa} \times \mathrm{m})$ at $\lambda=710 \mathrm{~nm}$. Moreover, the response to pressure is linear and shows small hysteresis in the investigated pressure range of 20 bars. The difference in sign of $K_{P}$ in the investigated fiber and in silica microstructured fibers of similar geometry is related to the different signs of the stress-optic coefficients in the two materials.

Acknowledgements: The work described in this paper was partially carried out with the support of the PHOSFOSproject ("Photonic Skins for Optical Sensing"), a Small/Medium-Scale Focused Project funded by the European Commission through the 7th ICT-Framework Programme and the Statutory Grant at Wroclaw University of Technology. M. K. Szczurowski, G. Satkiewicz-Barabach and W. Urbanczyk acknowledge support of the FNP Program "MISTRZ".

\section{REFERENCES}

[1] Kiesel S., Peters K., Hassan T., Kowalsky M., "Behaviour of intrinsic polymer optical fibre sensor for large-strain applications" Meas. Sci. Technol. 18, 3144-3154 (2007).

[2] Silva-López M., Fender A., MacPherson W. N., Barton J. S., Jones J. D. C., Zhao D., Dobb H., Webb D. J., Zhang L., Bennion I., "Strain and temperature sensitivity of a single-mode polymer optical fiber" Opt. Lett. 30, 3129-3131 (2005).

[3] Muto S., Suzuki O., Amano T., Morisawa M., "A plastic optical fibre sensor for real-time humidity monitoring" Meas. Sci. Technol. 14, 746-750 (2003).

[4] Yang X. H., Wang L. L. "Fluorescence pH probe based on microstructured polymer optical fiber" Opt. Express 15, 16478-16483 (2007).

[5] Xiong Z., Peng G. D., Wu B., Chu P.L., "Highly tunable Bragg gratings in single-mode polymer optical fibers" IEEE Photon. Technol. Lett. 11, 352-354 (1999).

[6] K. E.Carroll, C. Zhang, D. J. Webb, K. Kalli, A. Argyros, M. C. J. Large, "Thermal response of Bragg gratings in PMMA microstructured optical fibers" Opt. Express 15, 8844-8850 (2007).

[7] Dobb H., Webb D.J., Kalli K., Argyros A., Large M. C. J., Van Eijkelenborg M. A., "Continuous wave ultraviolet light-induced fiber bragg gratings in few- and single-mode microstructured polymer optical fibers" Opt. Lett. 30, 32963298 (2005).

[8] Szpulak M., Martynkien T., Urbanczyk W., "Effects of hydrostatic pressure on phase and group modal birefringence in microstructured holey fibers" Appl. Opt. 43, 4739-4745 (2004).

[9] Szpulak M., Martynkien T., Urbanczyk W., "Highly birefringent photonic crystal fibre with enhanced sensitivity to hydrostatic pressure", in Proceedings of 2006 8th International Conference on Transparent Optical Networks with 5th European Symposium on Photonic Crystals (Nottingham, UK, June 18-22, 2006) Vol. 4 / [Ed. M. Marciniak].

Piscataway, NJ : IEEE, cop. 2006, pp. 174-177.

[10] Primak W., Post D., "Photoelastic constants of vitreous silica and its elastic coefficient of refractive index" J. of Appl. Physics 30, 779-788 (1958).

[11] Waxler R. M., Horowitz D., Feldman A., "Optical and physical parameters of Plexiglas 55 and Lexan” Appl. Opt. 18, 101-104 (1979). 